complete value for ascorbic acid, but only measures the free acid. This amount is augmented by hydrolysis caused by heating. There may be present, also, an amount of reversibly oxidised ascorbic acid which is not measured by titration, unless it is first reduced by hydrogen sulphide, an observation recorded by Tillmans ${ }^{4}$ and others. Ascorbic acid in all these forms may be biologically active, but only one can be estimated by simple titration. Unlike these plant tissues, bovine adrenal tissue contains little bound ascorbic acid and none of the reversibly oxidised compound. Acid fruits, such as lemons, oranges and tomatoes, resemble adrenal tissue, in containing only free ascorbic acid.

School of Hygiene,

University of Toronto. March 21.

1 Biochem J., 27, 590; 1933.

2 Can. Chem. and Metallurgy, 18, $242 ; 1934$

${ }^{3}$ Biochem. J., 29, 275; 1935 .

'Z. Untersuch. Lebens., 68, 276 ; 1932.

\section{Extreme Infra-Red Investigation of Hindered Rotation in Water}

STRONG evidence for a quasi-crystalline structure in liquids has been furnished by X-ray analysis, the splitting of monochromatic light into a triplet by scattering, specific heat considerations, Raman effect ${ }^{1}$ and by an interpretation of viscosity ${ }^{2}$. Recently Debye $^{3}$ has succeeded in explaining several additional phenomena by assuming that not only do the molecules in a liquid vibrate under the influence of an intermolecular field about a centre of gravity which is slowly changing in space, but also that their free rotation is hindered. From two independent analyses, Debye concludes that in liquid water a rotation of the dipole moment through $90^{\circ}$ would produce a potential energy of about $10 \mathrm{kT}$.

The extreme infra-red spectrum of water vapour is composed of a multitude of lines extending beyond $500 \mu$ which correspond to the combination of pure rotational frequencies about the principal axes, having for moments of inertia ${ }^{4} 0.995,1.908$ and $2 \cdot 980 \times 10^{-40}$. The absorption of liquid water has been measured ${ }^{5}$ at $108 \mu$ and $313 \mu$ and led to the verification of Debye's dispersion formula for polar molecules. In Fig. 1 are shown the percentage of transmission and reflection of water between $52 \mu$ and $313 \mu$ (the filled circles refer to measurements made by Rubens ${ }^{5}$ ). From these data the absorption coefficient and index of refraction were calculated. The increasing values for the absorption coefficient and index of refraction from $90 \mu$ to $313 \mu$ are in agreement with the theory of dipole moments; however, an anomalously high absorption occurs below $90 \mu^{6}$. Considering the index of refraction to be continuously rising, in agreement with the simple dipole theory, this absorption produces an anomalous dispersion between $70 \mu$ and $90 \mu$, and is not due to internal vibrations of the water molecule (which are well known and are in the near infra-red), or to pure rotation bands (which have been measured in the vapour state throughout this region). This absorption has the appearance of a fundamental frequency and, following a suggestion of Prof. Debye's, might be due to a hindered rotation of the molecules.

Assuming the oscillations to be small and nearly harmonic, their frequency is given by $v=\frac{1}{2 \pi} \sqrt{\frac{\bar{K}}{I}}$, where $K$ is the torsional constant and $I$ the moment of inertia. Taking $\nu$ as $140 \mathrm{~cm}^{-1}$ (which, as might be expected, is not clearly defined) and considering rotation about the axis having a moment of inertia of $2 \cdot 980 \times 10^{-40}$ (which would be infra-red active), the torsional constant opposing free rotation amounts to $5 k T$. A frequency of $140 \mathrm{~cm}^{-1}$ corresponds to an energy of $0 \cdot 7 k T$; so there should be several quantum states for this hindered rotation. If we adopt the above value for the restoring torque, a hindered rotation about the axis having a moment of inertia of $0.998 \times 10^{-40}$ (which would also be infra-red active) would have its fundamental frequency at $240 \mathrm{~cm}^{-1}$ or $42 \mu$. This frequency lies close to the first overtone of the oscillation about the axis of greatest moment of inertia, so that especially strong absorption is expected near $40 \mu$; but our apparatus was not suitable for measuring below $52 \mu$.

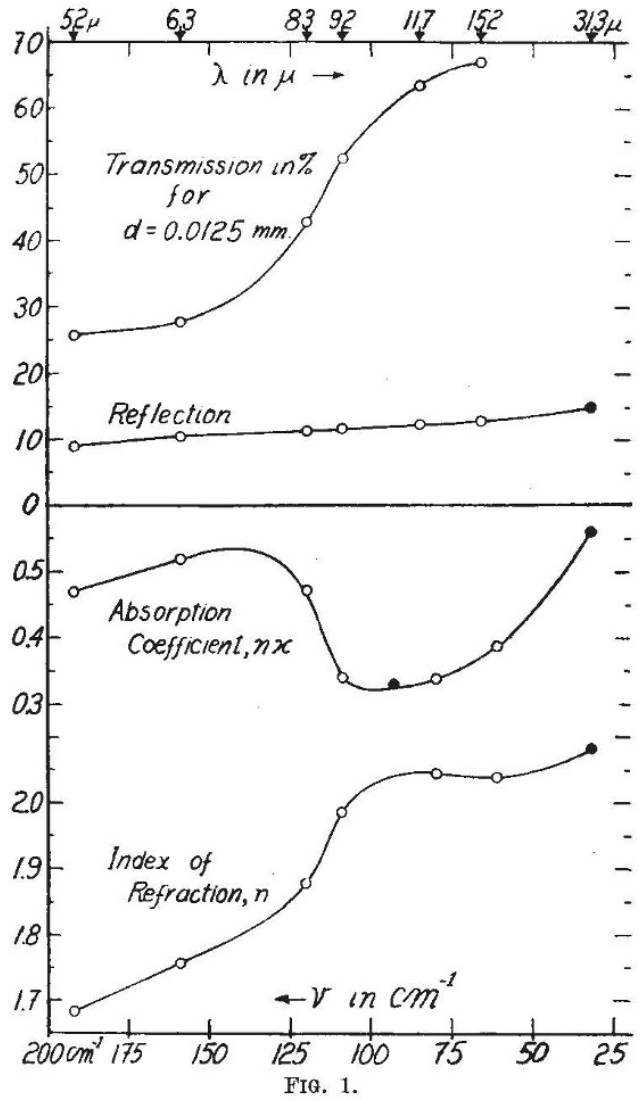

From our measurements, we conclude that the molecules in water are bound in a quasi-crystalline lattice and execute only partial rotation in agreement with Debye's hypothesis. Free rotation is hindered by a torsional constant of approximately $5 k T$, and the fundamental frequency for an oscillation about the axis of greatest moment of inertia occurs at about $140 \mathrm{~cm}^{-1}(70 \mu)$.

\section{Hawley Cartwright.}

Laboratory of Physical Chemistry,

Technical Faculty of the University, Brussels.

E. Gross and M. Vuks, NATURE, 135, $100 ; 1935$.

E. N. da C. Andrade, Phil. Mag., 17, 497, 689; 1934.

8 P. Debye, Phys. Z., 36, 100, 193 ; 1935.

R. Mecke, Z. Phys., 81, 313 ; 1933

${ }^{3} \mathrm{H}$. Rubens, Verh. d. d. phys. Ges., 17, 315; 1915.

${ }^{6} \mathrm{H}$. Rubens und E. Ladenburg, Verh. d. d. phys. Ges., 11, 16 ; 